\title{
Dynamic Navigation in Direction-Dependent Environments
}

\author{
Irina S. Dolinskaya * \\ Department of Industrial Engineering and Management Sciences, \\ Northwestern University, Evanston, IL 60208
}

\begin{abstract}
This chapter examines optimal path finding problems with direction, location and time dependent environments. The dependence of the cost function and path constraints on the location of the mobile agent and time creates the need for a dynamic navigation algorithm, capable of adjusting the path in real-time as more information about the environment becomes available. In addition, the direction-dependent nature of the environment results in an asymmetric cost function, which is not a metric and prohibits the use of more traditional and established approaches to solving optimal path finding problems. Moreover, the triangle inequality is often violated for the direction-dependent cost functions, further preventing the use of analysis and results developed for Euclidian shortest path problems. To add another dimension of reality to our model, we integrate the system dynamics and constrain feasible paths by maximum sharpness of a turn that a mobile agent can make.

The presented work delivers a more realistic optimal path-finding model while reducing the computational time required to find such a path. This is particularly important since real-time implementation is essential for our applications. In addition, many analytical results derived here provide insights into the structure of the problem, its objective function, and the optimal solution. These insights provide a closed-form solution to a large subset of problems where additional assumptions are applicable. For such problems, we easily construct the analytical solutions instead of implementing more involved, and often approximate, methods presented in the literature.

We describe the Optimum Vessel Performance in Evolving Nonlinear Wavefields Project that motivated our work, and deliver computational results to demonstrate the applicability and performance of our path-finding methods.
\end{abstract}

${ }^{*}$ This work was supported in part by the Office of Naval Research through the Multidisciplinary University Research Initiative (MURI) Optimum Vessel Performance in Evolving Nonlinear Wave Fields grant (N0001405-1-0537) and through the Autonomous Vehicle Dynamic Navigation System grant (N00014-11-1-0516). 


\section{Introduction}

Over the past few decades, researchers in a wide range of disciplines have been studying optimal path finding problems within a variety of applications. These approaches find an optimal way to traverse a complex medium or network under a diverse set of constraints and outside factors. For example, researchers in computational geometry and geographical information systems analyze the shortest paths defined by Euclidean distance and other metrics, often with the presence of polygonal obstacles and weighted homogeneous regions. Optimal robot routing problems incorporate the system's physical properties and constraints with the objective of finding the fastest or minimum energy-consumption paths over various terrain. In naval vessel path-finding and navigation, researchers integrate the vessel's hull structure and forces exerted by waves and wind to minimize travel time to a destination. Each aforementioned application adds complexity to the optimal path-finding problem, while integrating a number of assumptions in each scenario to make the problem more tractable. In this chapter, we relax a set of restrictive assumptions to broaden application of optimal path finding results to direction-dependent environments and to create an accurate and tractable models suitable for real-life implementation.

We study optimal path finding problems in a direction, location and time dependent environment. Since the objective of a problem depends on the actual application, we do not restrict our analysis to a specific objective function whenever possible. Throughout this chapter we discuss the problems of minimizing travel time, fuel consumption, and motions, as well as more general objective functions. The dependence of the cost function and path constraints on the location of the mobile agent, as well as time, creates the need for a dynamic navigation algorithm, capable of adjusting the path in real-time as more information about the environment becomes available. In addition, the direction-dependent nature of the environment results in an asymmetric cost function, which is not a metric and prohibits the use of more traditional and established approaches to solving optimal path finding problems. Moreover, the triangle inequality is often violated for the direction-dependent cost functions, further preventing the use of analysis and results developed for Euclidian shortest path problems. To add another dimension of reality to our model, we integrate the system dynamics and constrain feasible paths by maximum sharpness of a turn that a mobile agent can make.

The presented work delivers a more realistic optimal path-finding model while reducing the computational time required to find such a path. This is particularly important since realtime implementation is essential for our applications. In addition, many analytical results derived here provide insights into the structure of the problem, its objective function, and the optimal solution. These insights provide a closed-form solution to a large subset of problems where additional assumptions are applicable. For such problems, we easily construct the analytical solutions instead of implementing more involved, and often approximate, methods presented in the literature.

This chapter is an overview of a number of the author's prior publications (some co-authored with colleagues) on the said subject. It brings together our work within the various research fields into a single comprehensive dynamic navigation system for direction-dependent environments. For more detailed discussion of the presented work see [11, 12, 13, 14, 15]. 


\subsection{Motivation: Optimum Vessel Performance in Evolving Non- linear Wavefields Project}

Our work was motivated by an optimal vessel routing project entitled "Optimum Vessel Performance in Evolving Nonlinear Wavefields." This five-year project funded by the Office of Naval Research (ONR) Multidisciplinary University Research Initiative (MURI) grant is a collaboration with the Department of Naval Architecture and Marine Engineering and the Department of Industrial and Operations Engineering at the University of Michigan, the Applied Physics Laboratory at the University of Washington, and the Department of Electrical and Computer Engineering at The Ohio State University. Here, we provide a brief overview of the project and the research tasks of the teams involved. Throughout the chapter, we continually revisit this project to illustrate the real-life application of the developed methodology and results.

The goal of this project was to develop a system that can, in real-time, control the behavior of a vessel, based on real-time measurements and forecasts of the wavefield surrounding the vessel. Four major groups divided the project into the following parts based on the areas of expertise:

1. Real-Time Measurement of Ocean Wavefields. The first group of researchers develops and tests a coherent (Doppler) X-band radar for measurement of the ocean wavefield surrounding a moving or stationary vessel in real-time.

2. Short-Term Forecasts of Evolving Nonlinear Wavefields. The second team uses data collected by the radar to forecast the time-dependent evolution of the wavefield.

3. Time-Domain Computation of Nonlinear Ship Motions. Based on the forecast of the evolving wavefield, this group develops a numerical model to predict nonlinear ship motions in the multidirectional wavefield.

4. Dynamic Real-Time Path Optimization and Vessel Control. As part of the fourth team, we use the developed motion prediction model to evaluate the vessel speed, motions and other operability criteria conditional on a path chosen to traverse the forecasted wavefield. We then integrate this information into our optimal path finding algorithm to determine the most favorable path. An adaptive control system developed by our colleagues guides the vessel along the found optimal path as closely as possible.

This project considers a wide range of problems, and the objective varies depending on the specific application. Wavefield forecast can be used to predict time periods and areas of calm seas to ensure a safe landing onto an aircraft carrier or a successful launch-and-recovery operation in rough weather. Finding a path that minimizes ship motion is important for improving safety and comfort of the passengers on board. Minimizing travel time is crucial in emergency rescue missions and improves efficiency of the ship-to-ship or port-to-ship cargo transfer operations. Alternatively, finding a path that minimizes fuel consumption instead of the vessel's travel time is favorable for some naval transportation problems. Consequently, 
in our work we predominantly study a very general set of path finding problems, such that any one of the aforementioned applications can be addressed with our models.

To summarize, our objective as part of this project is to develop computationally efficient and numerically robust algorithms to solve path optimization problems in time-varying media. We are given information about the environment surrounding the vessel up to the radar visibility horizon and the dynamic restriction of the vessel: operability constraints such as probability of wet deck and maximum root mean square roll, and minimum turning radius constraining curvature of a feasible path. We incorporate this information to find an optimal path to a specified desired destination. It is important to note that the wave forecasting model developed as part of this project is precise, and the path finding problem is considered to be deterministic if the initial condition (i.e., the observed wavefield) is accurate.

\subsection{Literature Overview}

Existing literature details a wide variety of optimal path finding problems. While some work analyzes path finding in a location, and possibly, time dependent medium, others look at the scenarios of anisotropic (i.e., direction-dependent) environment. However, no previous research studies a generalized model that includes all aforementioned aspects of the environment into a single analysis. In this section, we present an overview of various areas of studies and applications that look at the optimal path finding problems as they relate to our work.

Geometric shortest path finding is a fundamental problem extensively studied in computational geometry. Mitchell's survey [32] gives a comprehensive overview of the current work conducted in this field. Most computational geometry research is restricted to finding an optimal path defined by Euclidean distance or other metrics, such as $L_{1}$-metric (the Manhattan distance) [30] and C-oriented paths [53]. Asymmetric direction-dependence is occasionally considered in the literature $[8,43]$, however the introduced anisotropy makes a strong assumption of distance function convexity which we relax in our analysis. The path-finding problems in a location-dependent environment examine the presence of polygonal obstacles $[2,21,24,29,31]$ and uniform-weighted regions [7, 33, 49]. On the other hand, all the problems studied in the field of computational geometry are predominantly static, and timedependence is not considered in these settings. It is important to note that Geographic Information Systems (GIS) is one of the primary application areas for computational geometry, and a number of papers published in GIS journals [9, 10, 48, 54] also discuss shortest path finding problems.

Optimal path finding research extends to other applications, such as robot, vessel, airplane and unmanned aerial vehicle routing. In each of these areas, researchers create the models specific to said application; unfortunately their analysis and results cannot be easily transferred to other problems. For example, the problem of computing an optimal path for a mobile robot considers friction and gravity forces for various regions of terrain, and then uses this direction and location dependent cost function to find a path that minimizes the total energy consumption of the robot $[23,44,45,50]$. Since surface contour does not change 
over time, this set of problems only considers path finding in a static environment.

Optimal vessel routing evaluates how waves and wind affect vessel speed and dynamics in finding an optimal path. For example, Philpott et al. [41] apply mathematical programming methods to create a yacht velocity prediction program that computes the vessel speed for a specified range of wind speeds and yacht headings. The resulting velocity prediction data is used in stochastic dynamic programming models to find the yacht's fastest path in uncertain weather $[1,39,40]$.

A significant amount of work assumes that the vessel speed function can be written analytically. This assumption allows researchers to invoke various methodologies from calculus of variations and optimal control theory to characterize an optimal path [18, 19, 26, 37, 38, 55]. However, researchers typically use a simplified form of the speed function in order to make the analysis more manageable. Our colleagues working on the Optimum Vessel Performance in Evolving Nonlinear Wavefields project have developed more accurate and involved models to evaluate vessel dynamics and wave evolution. From our experience of working on this project, it is clear that analytical functions cannot accurately describe the vessel movement through the waves, thus obliging us to look for alternative methods to solve the problem.

Airline industry researchers analyze how weather affects airplane path planning and air traffic management. For example, Nilim and his colleagues [34, 35] model the weather as Markov chains where storms have a certain probability of becoming the obstacles, thus preventing the airplanes from passing through those regions. Then, a path finding model identifies a path minimizing the expected travel time and dynamically reevaluates the path as more accurate information about the storms becomes available. In their work, Nilim et al. assume that the airplane has constant speed, consequently reducing the problem to a shortest path finding problem among stochastic obstacles.

Unmanned Aerial Vehicles (UAVs) have become widely employed in civilian and military applications over the past few years. The problem of optimal path finding for mini UAVs subjected to wind is similar in nature to the vessel routing problems, and has been extensively studied in recent years. The direction dependence of the speed function is introduced as a uniform wind vector field, which is added to a constant isotropic 'wind-free' velocity of the airplane $[3,27,28,36,52]$. It is important to note that the resulting speed function and the minimum turning radius of a feasible path have very distinct structures, and more specifically, the properties of a convex speed polar plot and trochoidal path [46]. In our work, we observe that direction-dependent speed often implies the direction-dependent nature of the minimum turning radius, and we address such problems for the generalized direction-dependent speed functions and path curvature restrictions.

\subsection{Chapter Outline}

This chapter is organized as follows. We begin our analysis by studying optimal path finding problems in a direction-dependent, time and space homogeneous environment, which is presented in Section 2. First, we find closed form solutions for the problems with obstacle-free domain while neglecting the minimum turning radius constraint (Section 2.1). Then, we em- 
ploy our findings and adapt a visibility graph search method of computational geometry to an anisotropic environment, delivering an algorithm that finds an optimal obstacle-avoiding path in a direction-dependent medium.

Section 2.2 extends our analysis of path finding in an anisotropic, time and space homogeneous environment to a set of problems where path curvature is constrained by a very general direction-dependent minimum turning radius function. We demonstrate the problem's controllability, prove existence of an optimal path, and invoke techniques from optimal control theory to derive a necessary condition for optimality. Further analysis characterizes an optimal path and delivers an algorithm that facilitates the implementation of the presented results.

The assumption of time and space homogeneity is relaxed in Section 3, where we develop a dynamic programming model to find an optimal path in a location, direction and time dependent environment. The results from the preceding section are integrated into the model to improve its accuracy, efficiency and run-time. The path finding model addresses limited information availability (Section 3.1), control-feasibility (Section 3.2) and computational demands of a time-dependent environment (Section 3.3). The step-by-step path finding algorithm (Section 3.4) and its application to the Optimum Vessel Performance in Evolving Nonlinear Wavefields project (Section 3.5) are also presented in this section. In Section 4 we discuss how to extend our analysis to optimal path finding problems for cost functions other than travel time. The chapter concludes with Section 5 summarizing the results, contributions and future directions of our work.

\section{Optimal Path Finding in Direction-Dependent, Time and Space Homogeneous Environment}

We begin our study of dynamic navigation in direction-dependent (anisotropic) environment by focusing our attention on the effects the direction-dependence has on path optimality. As discussed in the introduction, the anisotropic cost function is not a metric, since traveling along the straight line path from $a$ to $b$ does not necessarily incur the same cost as traveling along the reversed straight line path from $b$ to $a$. In addition, the triangle inequality might not hold true, and the straight line path is not always optimal. In this section, we assume time and space homogeneity of the environment and extend existing results from computational geometry and control theory to the direction-dependent medium. In the following Section 3, these results are integrated into the optimal path finding algorithm for a direction, location and time dependent environment.

\subsection{Optimal Path Finding Without Turning Radius Constraint}

We address the fastest-path finding problems with anisotropic environment where the speed function, $V(\theta)$, is direction-dependent, such as in the case when ocean waves, winds or slope of the terrain affect agent's motions. Our objective is to find a path from a given starting 
location to a given target point that minimizes total travel time of the mobile agent. We first solve this problem in an obstacle-free domain and then integrate polygonal obstacles restricting the set of feasible paths. It is important to note that while we demonstrate our analysis and results for the fastest-path finding problem, the discussion can be easily extended to other additive direction-dependent cost functions (e.g., fuel consumption). This section presents a brief overview of our joint work with R.L. Smith; for more detailed analysis and results see $[11,15]$.

Let $\mathcal{S}(V(\theta)) \subseteq \Re^{2}$ denote the set of points enclosed by the polar plot of a speed function $V(\theta)$ centered in the origin point $O=(0,0) \in \Re^{2}$. From the definition it follows that $\mathcal{S}(V)$ contains all the points that the mobile agent can reach from point $O$ along the straight line path within a single unit of time. We let $\tau(V, x)$ for $x \in \Re^{2}$ denote the travel time along the straight line path from $O$ to $x$ for the speed function $V(\theta)$. Note, for all $x \in \mathcal{S}(V)$, $\tau(V, x) \leq 1$.

First, consider a special case when $\mathcal{S}(V(\theta))$ is a convex set. Then, properties of the Minkowski functional [25] establish that the straight line path is the fastest path between a pair of points for the speed function $V(\theta)$, and that the triangle inequality holds true. In the case when the speed polar plot for $V(\theta)$ is not convex, we consider the augmented speed function $V_{a}(\theta)$, such that its polar plot is equal to the convex hull of the original speed polar plot, i.e., $\mathcal{S}\left(V_{a}(\theta)\right)=\operatorname{Conv}\left(\mathcal{S}(V(\theta))\right.$ ) (see Figure 1). Then, the speed function $V_{a}$ corresponds to a convex speed polar plot and the straight line optimal paths. Furthermore, $V(\theta) \leq V_{a}(\theta)$ for all $\theta$, and the travel time along an optimal (straight line) path with the speed function $V_{a}$ is a lower bound on the optimal travel time between the same two points with the speed function $V(\theta)$. That is, $\tau\left(V_{a}, x\right)$ is the lowest possible travel time between points $O$ and $x$ for a mobile agent with the speed function $V(\theta)$.
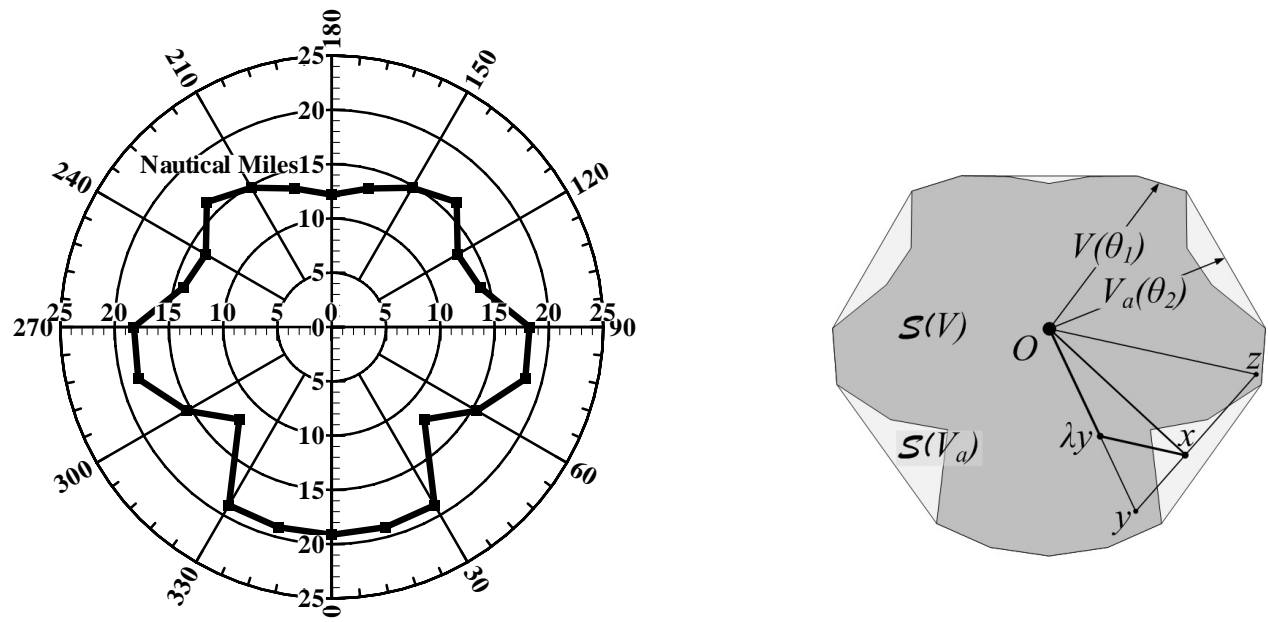

Figure 1: An example of speed polar plot for the S-175 containership [13] and corresponding application of our analysis.

Observe that, since $\mathcal{S}\left(V_{a}\right)$ is the convex hull of $\mathcal{S}(V)$, every point in $\mathcal{S}\left(V_{a}\right)$ can be expressed as a convex combination of two points in $\mathcal{S}(V)$. In other words, $\forall x \in \mathcal{S}\left(V_{a}\right)$, there exist 
$y, z \in \mathcal{S}(V)$ and $\lambda \in[0,1]$ such that $x=\lambda y+(1-\lambda) z$. Then, every point $x \in \mathcal{S}\left(V_{a}\right)$ can be reached following a piecewise linear path from $O$ to $\lambda y$ and then from $\lambda y$ to $x$. Finally, observe that $\tau(V, y)=\tau\left(V_{a}, y\right), \tau(V, z)=\tau\left(V_{a}, z\right)$ and the travel time along the described piecewise linear path with speed function $V(\theta)$ is equal to $\tau\left(V_{a}, x\right)$, thus establishing its optimality. See Figure 1 for an illustration of the presented analysis for the test vessel used in the Optimum Vessel Performance in Evolving Nonlinear Wavefields project.

We have established that in an obstacle-free domain, an optimal path for an arbitrary anisotropic speed function is piecewise linear with at most one way-point. Next, we employ these findings to the problems that consider the presence of polygonal obstacles. For the case when the speed function corresponds to a convex polar plot, the straight line path is a fastest path in $\Re^{2}$. Therefore, fastest-path finding in the presence of polygonal obstacles can be restricted to a modified visibility graph, similarly to Euclidian shortest path finding problems [2, 24], (which essentially searches for fastest path among taut-string paths between starting and target points). We construct the visibility graph by defining the set of vertices to be all the vertices of the polygonal obstacles, as well as the starting and target points. The set of edges of our visibility graph consists of all the straight line edges interconnecting the defined vertices that do not intersect any of the obstacles. And finally, the cost function for each edge is set to be the travel time along the link characterized by $\tau(V,$.$) function. Then,$ a minimum cost path in the constructed graph corresponds to the fastest obstacle-avoiding path for our original problem.

The triangle inequality might not hold true for a general direction-dependent speed function $V(\theta)$. In such case, an augmented speed function, $V_{a}(\theta)$, corresponding to the convex hull of the original speed polar plot is used to find a lower bound on the minimum travel time for our problem. We construct a visibility graph as discussed above for the augmented speed function, $V_{a}(\theta)$. We then implement the piecewise linear results presented for the obstaclefree domain along each edge of the optimal path in this graph (see Figure 2). Thus, we construct an obstacle-avoiding path that achieves this lower bound, implying its optimality.

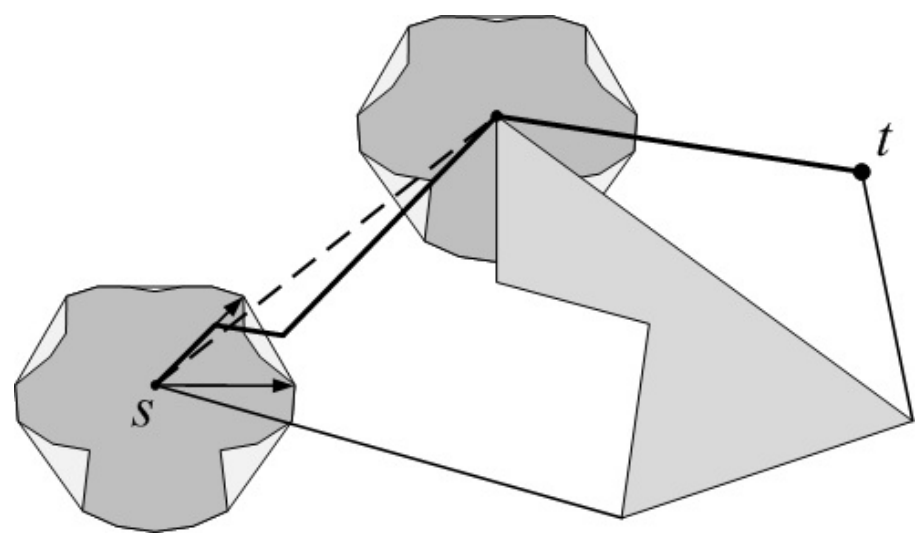

Figure 2: An example of an obstacle-avoiding fastest path for the S-175 containership. 


\subsection{Optimal Path with Bounded Curvature in an Anisotropic Medium}

Our initial analysis described above shows that an optimal path in an anisotropic time and space homogeneous environment has a piecewise-linear structure. Unfortunately, the instantaneous heading change required to follow a piecewise-linear path is infeasible for most applications, including the navigation of aerial, surface and naval vehicles. For these problems, the control system of an agent constrains the set of feasible paths and these restrictions must be integrated into the optimal path finding process. We introduce a direction-dependent minimum turning radius function $R(\theta)$ that constrains the curvature of a feasible path for a vehicle with the heading direction $\theta$ and find an optimal path with bounded curvature in an anisotropic time and space homogeneous environment. More detailed analysis and results of the work presented here can be found in $[11,14]$.

The problem's objective is to find a fastest path that starts at the initial point $\left(x_{s}, y_{s}\right)$ and heading angle $\theta_{s}$, ends at a destination point $\left(x_{t}, y_{t}\right)$ with a predetermined final heading $\theta_{t}$, and has a curvature bounded by a specified minimum turning radius function $R(\theta)$. Most published work that discusses fastest-path finding problems with bounded curvature (e.g., $[4,5,17,47,51])$ assumes constant speed and minimum turning radius. When the presence of direction-dependence is introduced in the existing literature, the resulting speed and minimum turning radius functions maintain specific structures and properties. We analyze the problems in the anisotropic media where both the agent's speed and minimum turning radius are described by generalized direction-dependent functions. The direction-dependent nature of this problem implies the same asymmetry of a travel time function as discussed in preceding sections. Additionally, the non-constant turning radius results in complex sharpest turn curves, as opposed to a circle, which is an essential part of an optimal path for the isotropic problems. These facts make the task of extending the problem of optimal path finding with minimum curvature to the direction-dependent case a significant challenge.

Let $(x(t), y(t), \theta(t)) \in \Re^{2} \times S^{1}$ denote the vehicle configuration at time $t \in[0, T]$, where $(x(t), y(t))$ are the coordinates of the mobile agent position and $\theta(t)$ is its heading angle with respect to the $x$ axis. We set the system steering controller $u(t):[0, T] \rightarrow[-1,1]$ to represent the rate of change of the vehicle heading at time $t$. Then, we can write our fastest path finding problem as the following differential system:

$$
\begin{aligned}
& \min _{u} T \\
\text { subject to } \dot{x}= & V(\theta) \cos (\theta), \\
\dot{y}= & V(\theta) \sin (\theta), \\
\dot{\theta}= & \frac{V(\theta)}{R(\theta)} u,
\end{aligned}
$$

with the boundary conditions:

$$
\begin{aligned}
(x(0), y(0), \theta(0)) & =\left(x_{s}, y_{s}, \theta_{s}\right), \\
(x(T), y(T), \theta(T)) & =\left(x_{t}, y_{t}, \theta_{t}\right) .
\end{aligned}
$$


We demonstrate the problem's controllability by reducing our problem to Dubins car problem [17], which has been established to be controllable [51]. Next, we prove the existence of an optimal path using Filippov's Theorem [20]. Then, we employ optimal control theory and Pontryagin's Principle [42] and derive the necessary condition for optimality that states that any optimal path is the concatenation of the arcs with minimum turning radius $R(\theta)$ and the straight line segments. Note that this condition for optimality holds true for the very generalized anisotropic speed function $V(\theta)$ and minimum turning radius function $R(\theta)$. Further analysis delivers the detailed characterization of an optimal path structure.

Theorem 1 (From [14]). There exists an optimal path from an initial configuration $\left(x_{s}, y_{s}, \theta_{s}\right)$ to a target configuration $\left(x_{s}, y_{s}, \theta_{s}\right)$ such that it is a portion of a path of type $\mathcal{C S C S C}$ where $\mathcal{C}$ denotes a sharpest turn and $\mathcal{S}$ a straight line.

For the case when the speed polar plot is convex we have more specific characterization.

Theorem 2 (From [14]). When the movement along a path is characterized by a speed function with a convex polar plot, an optimal path from $\left(x_{s}, y_{s}, \theta_{s}\right)$ to $\left(x_{t}, y_{t}, \theta_{t}\right)$ is of the form $\{\mathcal{C}, \mathcal{C}, \mathcal{C}\}$, or $\{\mathcal{C}, S, \mathcal{C}\}$, where $\mathcal{C}$ denotes a sharpest turn curve and $S$ denotes a straight line segment. It is implied that a path of the form $\{\mathcal{C}, \mathcal{C}, \mathcal{C}\}$ alternatively switches between left-hand and right-hand sharpest turn curves.

See [14] for the detailed proofs of these theorems and an algorithm that facilitates the implementation of the results.

\section{Dynamic Programming Modeling for Optimal Path Finding in a Direction, Location and Time Depen- dent Environment}

In the preceding section, an optimal path in a time and space homogeneous directiondependent environment was found; in other words, the cost function and constraints are assumed to be independent of the time and location of an agent. Here, we relax the assumption of homogeneity and discuss a dynamic programming model for optimal path finding in a direction, location and time dependent environment. This section is a brief overview of our work discussed in $[11,12]$.

\subsection{Limited Visibility Horizon}

Current technological advancements in real-time data collection and forecasting call for an explicit incorporation of the available information into the decision making process. Innovative on-board sensors, such as a Doppler radar in the Optimum Vessel Performance in Evolving Nonlinear Wavefields project, collect information about the surrounding environment in real-time. The optimal path finding model presented here makes use of the gathered 
information. It is important to acknowledge that physical sensors have a limited visibility horizon and cannot gather information about the medium beyond a specified distance, which is often closer than the location of the target point. To address this restriction, our model presumes to have complete information about the environment (i.e., cost function and constraints) within the radar visibility horizon (denoted by $R_{H}$ ) and limited information beyond that horizon (see Figure 3).

Inside the radar visible region (i.e., region within $R_{H}$ distance from the current location of the mobile agent) the medium is completely characterized by the available information. This allows us to construct a detailed dynamic programming model (to be described below) to evaluate optimal paths to all the points on the boundary of the visible region. Due to the limited information available to us about the environment beyond $R_{H}$, we approximate that region of the medium by a stationary distribution characterized by a global parameter. For example, in the case of vessel routing, a parameter called sea state characterizes the distribution of waves to be encountered for a period of a few hours. As a result, we assume the environment beyond $R_{H}$ is time and space homogeneous for the duration of the trip. This assumption facilitates the use of our earlier results (see Section 2) to find an optimal path from each point on the boundary of the radar visible region to the target point. Note that as the mobile agent travels along a path, the radar visible region moves with it, and the optimal path is reevaluated in real-time incorporating the new information.

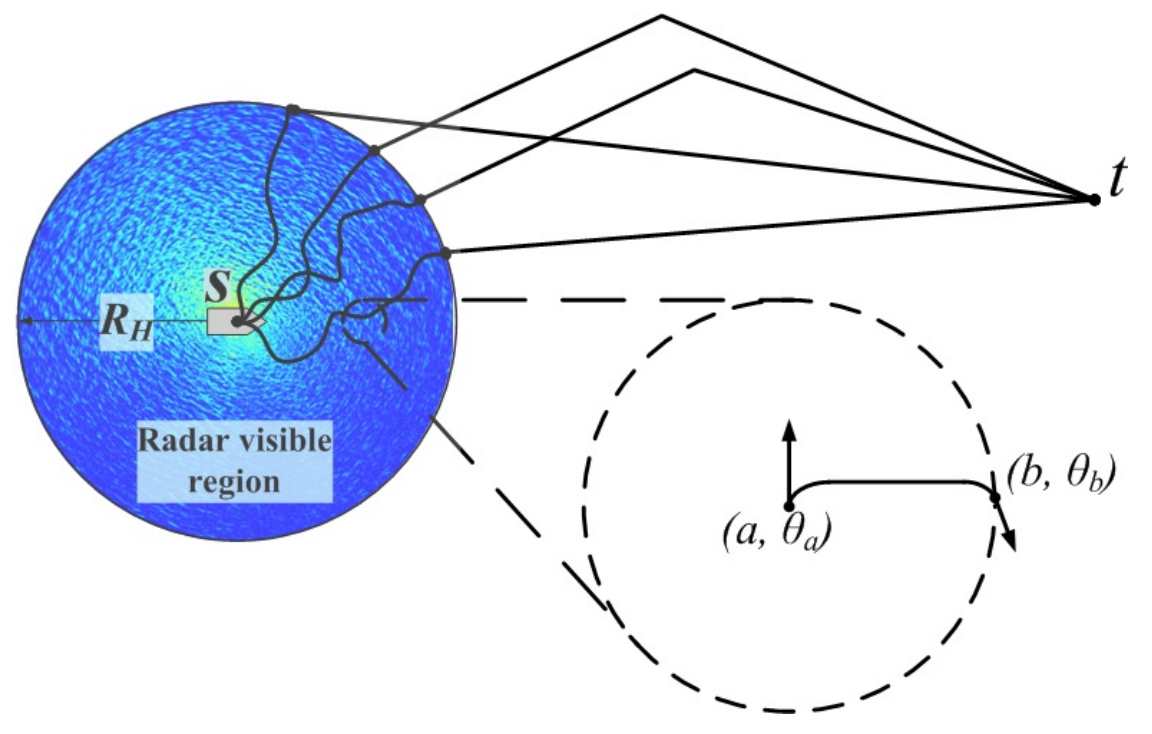

Figure 3: Dynamic Programming model finds the fastest paths to the points on the visibility horizon, then results for time and space homogeneous medium are used to find the best paths to continue.

\subsection{System Dynamics Restrictions}

The traditional dynamic programming (DP) path finding models discretize the path domain into a set of way-points and find an optimal ordered set of way-points to traverse from the 
starting point to a target location. Since the paths between a consecutive pair of way-points is assumed to be a straight line segment, the resulting optimal path is piecewise linear. However, in a number of real-life applications, such as navigation of aerial, ground and naval vehicles, piecewise linear paths are not feasible, since the curvature of a feasible path is constrained by a minimum turning radius function. Instead of the traditional approach of addressing the optimal path finding and path-following aspects of the problem separately, we integrate the system's operability and dynamics constraints into an optimization model resulting in a control-feasible solution.

We develop a dynamic programming model to be implemented inside the visible region to find an optimal path to all the points on the boundary of this region (i.e., $R_{H}$ distance away from the agent's current location). We discretize the path domain into a set of waypoints $l$ distance away from each other, and the environment of the $l$-radius neighborhood surrounding each way-point is assumed to be time and space homogeneous. We set our dynamic programming state to include the location (i.e., way-point), as well as the heading angle of the mobile agent at the way-point. Then, at each state, the dynamic programming model decides on the next way-point and the heading angle with which to arrive at that point. Since the environment between the two consecutive way-points is assumed to be time and space homogeneous, we use our results for optimal path with bounded curvature presented in Section 2.2 to find the fastest path and corresponding travel time between the DP states (see Figure 3).

\subsection{Computational Demand of a Time-Dependent Environment}

Due to the time dependency of the environment and cost function, it is not necessarily optimal to arrive at each intermediate way-point of a path as soon as possible. To account for this fact, a time variable is traditionally added to the dynamic programming state in order to keep track of all possible times at which we might arrive, and consequently leave, a given point. This additional variable increases the number of DP states to be considered by orders of magnitude. At the same time, computational demand and run-time of the optimal path finding model is of particular significance to timely utilization of the available real-time information in a decision making process. We developed an alternative formulation of the dynamic programming functional equation, which allows us to eliminate the time variable from the state space, thus significantly reducing the computation time of our algorithm.

Our approach is built on work by Dreyfus [16], who was the first one to demonstrate that in the case when unlimited waiting in the nodes is allowed, the dynamic fastest path problem can be solved using Dijkstra's algorithm as efficiently as in the case of a static network. Dreyfus redefines the cost function $d_{i j}\left(t_{i}\right)$ (the cost of traveling from node $i$ to node $j$ when leaving node $i$ at time $t_{i}$ ), so that "if travel schedules are such that a delay before departure decreases the time of arrival, $d_{i j}\left(t_{i}\right)$ represents the elapsed time between time $t_{i}$ and the earliest possible time of arrival." This alternative definition of $d_{i j}\left(t_{i}\right)$ ensures the validity of the consistency condition (established in [22]) and facilitates a straight forward dynamic programming formulation of the problem, where DP state only stores the current location in the network. However, stopping at the waypoints is impractical or infeasible for many 
applications. For example, an airplane cannot stop in mid-air to wait for a storm to pass by. Similarly, it is not practical for a large vessel to come to a complete stop before continuing its travel. Consequently, we do not allow stopping or waiting in our model. Instead, we extend Dreyfus' approach to a path finding model permitting voluntarily speed reduction (i.e., slow down) along a path.

Let $\tau\left(a, \theta_{a}, b, \theta_{b}, t_{a}\right)$ denote the vessel travel time along the fastest path with bounded curvature from point $a$ to point $b$ starting at heading angle $\theta_{a}$ at time $t_{a}$ and arriving at heading angle $\theta_{b}$. Define $g\left(a, \theta_{a}\right)$ to be the minimum travel time from the starting position $\left(s, \theta_{s}\right)$ to point $a$, arriving at $a$ with the heading angle $\theta_{a}$. Recall that the distance between two consecutive way-points is denoted by a fixed parameter $l$. Then, we formulate the following DP functional equation,

$$
g\left(b, \theta_{b}\right)=\left\{\min _{\left\{a, \theta_{a}:\|b-a\|=l\right\}}\left\{g\left(a, \theta_{a}\right)+\min _{\Delta_{t}}\left(\Delta_{t}+\tau\left(a, \theta_{a}, b, \theta_{b}, g\left(a, \theta_{a}\right)+\Delta_{t}\right)\right)\right\},\right.
$$

where $\|\cdot\|$ is Euclidean norm. By setting the initial condition $g\left(s, \theta_{s}\right)=0$ and iteratively applying the functional equation (1), we find fastest paths to all the points on the boundary of the visible region. Note that $\Delta_{t}$ denotes the vessel 'delay' at a given way-point before continuing its travel. Thus, the solution of our dynamic programming model returns an ordered set of optimal way-points and optimal delay time at each of those points. Since we assume that waiting (i.e., delay) is not permitted in the intermediate points of a path, we instead intentionally slow down the mobile agent to guarantee that its arrival time to a way-point coincides with the optimal time to depart it.

\subsection{Fastest Path Finding Algorithm (adopted from [12])}

Step 1. Apply results from Section 2.2 to compute the values of $\tau\left(a, \theta_{a}, b, \theta_{b}, t_{a}\right)$ for all inputs where $\|a-s\| \leq R_{H},\|b-s\| \leq R_{H}$ and $\|a-b\|=l$.

Step 2. Apply Dijkstra's algorithm to the DP recursive equation (1) to find the fastest paths from $\left(s, \theta_{s}\right)$ to a discretized set of points on the visibility horizon $R_{H}$.

Step 3. Apply results presented in Section 2.1 or Section 2.2 to find the fastest paths from the points on the visibility horizon to the target state $\left(t, \theta_{t}\right)$.

Step 4. Find the discretized point on the visibility horizon that has the smallest sum of the corresponding travel times found in Step 2 and Step 3. A fastest path passing through such point is the optimal path.

Step 5. For the optimal path found in Step 4, adjust the speed for each arc as discussed in Section 3.3 to ensure optimal arrival to each intermediate waypoint. 


\subsection{Numerical Results}

To demonstrate the applicability and performance of our path-finding methods, we conducted computational experiments for the Optimum Vessel Performance in Evolving Wavefield project. The test runs are simulated for an S-175 containership in the Sea State 6.5 wavefield (corresponding to the mean wave height of the one third highest waves of 7 meters). We compared the travel time of the found optimal path to those of the straight line path and one way-point path, which we established to be optimal for time and space homogeneous environment. We observed the average savings varying between $4 \%$ and $6 \%$, with up to $9.7 \%$ saving. However, these estimations are very conservative due to a number of data limitations and restricted maneuverability of the 175-meter long vessel. When we reduce the minimum turning radius of the test vessel by half, we report an average improvement in travel time of $12.5 \%$. See $[11,12]$ for complete discussion of our numerical results.

\section{Optimal Path Finding for a Cost Function Other Than Travel Time}

Throughout this chapter, we discuss an optimal path finding problem with the objective of minimizing the agent's travel time. We often mention that our analysis and results can be directly extended to problems minimizing other cost functions. In many applications, we face an optimal path finding problem with alternative objective functions. For example, in the case of the optimum vessel performance in the evolving wavefield project, in addition to finding a fastest path, we are interested in minimizing root mean squared (RMS) motions, such as roll (2) and other measures of the path 'quality'.

$$
R M S_{\text {Roll }}=\sqrt{\frac{\phi_{1}^{2}+\ldots+\phi_{n}^{2}}{n}} .
$$

However, the extension of our analysis and presented path finding model is not straightforward for dynamic networks and path finding in a time-dependent environment. In this section we discuss how an optimal path finding algorithm changes when the objective function is different from travel time.

The problem of minimizing cost in a dynamic network is briefly discussed in the literature. For example, Chabini [6] looks at the minimum cost functions where travel time functions $d_{i j}\left(t_{i}\right)$ and cost functions $c_{i j}\left(t_{i}\right)$ are time-dependent. He extends a backward DP formulation of the fastest path problems to this minimum cost path finding problem. Chabini assumes that $d_{i j}\left(t_{i}\right)$ and $c_{i j}\left(t_{i}\right)$ are constant for any time greater than some specified value, resulting in a static problem. This static problem solution is then used as the boundary condition for the dynamic programming formulation of the problem.

The difference between our earlier analysis of the fastest-path finding problems and modeling of a problem with a general cost function is that we cannot eliminate the time variable from 
the dynamic programming state space (see Section 3.3 and equation (1)). Therefore, we have to set the DP state to be $\left(a, \theta_{a}, t_{a}\right)$ and consider all possible times of arrival at a given waypoint. Consequently, the resulting DP model delivers a classical functional equation and a straightforward application of Dijkstra's method or Chabini's approach to find an optimal path.

We also note that the cost function has to be additive to apply the standard dynamic programming recursive equation. However, the model can be adjusted to other objective functions. For example, the averaging measures of path quality, like RMS roll, can be implemented by fixing a constant number of arcs for all considered feasible paths, or by adding a variable keeping record of the number of arcs traveled to the dynamic programming state space. Alternately, since in the Optimum Vessel Performance in Evolving Wavefield project we are interested in minimizing roll experienced by a vessel without significant increase in trave time, we set our dynamic programming model to minimize the additive function $\phi_{1}^{2}+\ldots+\phi_{n}^{2}$ instead of the $R M S_{\text {Roll }}$ defined in equation (2). This, in turn, allows the model to capture the trade-off between travel time and RMS roll of a given path. In our forthcoming work we further explore this and other similar problems.

\section{Conclusion}

This chapter discusses optimal path finding in a direction, location and time dependent environment. We deliver a computationally-efficient path finding algorithm with a sufficiently small run-time for real-time implementation. A traditional dynamic programming path finding model makes a number of restrictive assumptions that jeopardize its applicability to real-life problems. Alternatively, we present a model that integrates and addresses a set of limiting aspects previously neglected in the literature:

- Our dynamic programming (DP) path finding model integrates a limited visibility horizon and accounts for the lack of detailed information about a medium beyond a certain distance from the mobile agent's current location.

- The presented DP model finds a smooth and control-feasible fastest path by integrating the systems dynamics into the optimization process.

- By integrating the agent's controller (speed) into the decision space of the algorithm, the resulting model eliminates a time variable from the dynamic programming state space and improves efficiency and run-time of our model.

A number of special case problems corresponding to the assumption of a time and space homogeneous environment are solved analytically. These results deliver a significant contribution to the study of anisotropic (direction-dependent) problems.

We are currently working on integrating the additional system constraints, such as bounded acceleration and deceleration, into the optimal path finding model described in Section 2.2 in order to improve its accuracy and applicability. We also plan to integrate uncertainty 
associated with data-collection and forecasting errors of the future environment. The goal of our ongoing work is to continue the study of integration of real-time data collection into the optimization models, especially with application to unmanned systems.

\section{Acknowledgment}

The author would like to thank Robert L. Smith from the University of Michigan for his helpful guidance and discussions.

\section{References}

[1] Allsopp, T., Mason, A., And Philpott, A. Optimal sailing routes with uncertain weather. In Proceedings of The 35th Annual Conference of the Operational Research Society of New Zealand (December 2000), pp. 65-74.

[2] Alt, H., And Welzl, E. Visibility graphs and obstacle-avoiding shortest paths. $Z$. Oper. Res. 32, 3-4 (1988), 145-164.

[3] Bakolas, E., and Tsiotras, P. Time-optimal synthesis for the Zermelo-MarkovDubins problem: The constant wind case. In Proceeding of 2010 American Control Conference (Baltimore, MD, June 30 - July 2, 2010).

[4] Boissonnat, J.-D., CÉrÉzo, A., And Leblond, J. Shortest paths of bounded curvature in the plane. Journal of Intelligent and Robotic Systems, 1-2, 5-20.

[5] Bui, X.-N., Souères, P., Boissonnat, J.-D., And Laumond, J.-P. Shortest path synthesis for Dubins non-holonomic robot. In Proceedings of the 11th IEEE Internatational Conference on Robotics Automation (1994), pp. 2-7.

[6] Chabini, I. Discrete dynamic shortest path problems in transportation applications: Complexity and algorithms with optimal run time. Transportation Research Record 1645 (1998), 170-175.

[7] Cheng, S.-W., Na, H.-S., Vigneron, A., And Wang, Y. Approximate shortest paths in anisotropic regions. SIAM J. Comput. 38, 3 (2008), 802-824.

[8] Chew, L. P., And Drysdale, III, R. L. S. Voronoi diagrams based on convex distance functions. In $S C G$ '85: Proceedings of the first annual symposium on Computational geometry (New York, NY, USA, 1985), ACM, pp. 235-244.

[9] Collischonn, W., And Pilar, J. V. A direction dependent least-cost-path algorithm for roads and canals. Int. J. Geogr. Inf. Sci. 14, 4 (2000), 397-406. 
[10] de Floriani, L., Magillo, P., And Puppo, E. Applications of computational geometry to geographic information systems. In Handbook of computational geometry. North-Holland, Amsterdam, 2000, pp. 333-388.

[11] Dolinskaya, I. S. Optimal Path Finding in Direction, Location and Time Dependent Environments. PhD thesis, University of Michigan, 2009.

[12] Dolinskaya, I. S. Optimal path finding in direction, location and time dependent environments. Under Review (2011).

[13] Dolinskaya, I. S., Kotinis, M., Parsons, M. G., and Smith, R. L. Optimal short-range routing of vessels in a seaway. Journal of Ship Research 53, 3 (September 2009), 121-129.

[14] Dolinskaya, I. S., And Maggiar, A. Time-optimal trajectories with bounded curvature in anisotropic medium. Under Review.

[15] Dolinskaya, I. S., And Smith, R. L. Path planning in an anisotropic medium. Under Review.

[16] Dreyfus, S. E. An appraisal of some shortest-path algorithms. Operations Research 17, 3 (1969), 395-412.

[17] Dubins, L. E. On curves of minimal length with a constraint on average curvature, and with prescribed initial and terminal positions and tangents. Amer. J. Math. 79 (1957), 497-516.

[18] Faulkner, F. D. A general numerical method for determining optimum ship routes. Navigation 10, 2 (1963), 143-148.

[19] Faulkner, F. D. Numerical methods for determining optimum ship routes. Navigation: Journal of The Institute of Navigation 10, 4 (Winter 1963), 351-367.

[20] Filippov, A. F. On certain questions in the theory of optimal control. Journal of the Society for Industrial and Applied Mathematics, Series A: Control 1, 1 (1962), 76-84.

[21] Kapoor, S., Maheshwari, S. N., and Mitchell, J. S. B. An efficient algorithm for Euclidean shortest paths among polygonal obstacles in the plane. Discrete Comput. Geom. 18, 4 (1997), 377-383.

[22] Kaufman, D. E., And Smith, R. L. Fastest paths in time-dependent networks for intelligent vehicle-highway systems application. IVHS Journal 1, 1 (1993), 1-11.

[23] Lanthier, M., Maheshwari, A., And Sack, J.-R. Shortest anisotropic paths on terrains. In Automata, languages and programming (Prague, 1999), vol. 1644 of Lecture Notes in Comput. Sci. Springer, Berlin, 1999, pp. 524-533.

[24] Lozano-Pérez, T., And Wesley, M. A. An algorithm for planning collision-free paths among polyhedral obstacles. Commun. ACM 22, 10 (1979), 560-570. 
[25] Luenberger, D. G. Optimization by vector space methods. John Wiley \& Sons Inc., New York, 1969.

[26] Marks, W., Goodman, T. R., W. J. Pierson, J., Tick, L. J., and VassilopouLOS, L. A. An automated system for optimum ship routing. Transactions - The Society of Naval Architects and Marine Engineers 76 (1968), 22-55.

[27] McGee, T. G., Spry, S., And Hedrick, J. K. Optimal path planning in a constant wind with a bounded turning rate. In Proceedings of the AIAA Conference on Guidance, Navigation and Control (Ketstone, Colorado, August 2006).

[28] McNeely, R. L., Iver, R. V., And Chandler, P. R. Tour planning for an unmanned air vehicle under wind conditions. Journal of Guidance, Control, and Dynamics 30, 5 (September-October 2007), 1299-1306.

[29] Mitchell, J. S. B. A new algorithm for shortest paths among obstacles in the plane. Ann. Math. Artificial Intelligence 3, 1 (1991), 83-105.

[30] Mitchell, J. S. B. $L_{1}$ shortest paths among polygonal obstacles in the plane. Algorithmica 8, 1 (1992), 55-88.

[31] Mitchell, J. S. B. Shortest paths among obstacles in the plane. Internat. J. Comput. Geom. Appl. 6, 3 (1996), 309-332.

[32] Mitchell, J. S. B. Geometric shortest paths and network optimization. In Handbook of computational geometry. North-Holland, Amsterdam, 2000, pp. 633-701.

[33] Mitchell, J. S. B., And Papadimitriou, C. H. The weighted region problem: finding shortest paths through a weighted planar subdivision. J. Assoc. Comput. Mach. 38, 1 (1991), 18-73.

[34] Nilim, A., And Ghaoui, L. E. Algorithms for air traffic flow management under stochastic environments. In Proceedings of American Control Conference (July 2004), vol. 4, pp. 3429-3434.

[35] Nilim, A., Ghaoui, L. E., Hansen, M., and Duong, V. Trajectory-based air trafic management (tb-atm) under weather uncertainty. In Proceedings of the Fourth International Air Traffic Management R\&D Seminar ATM (Santa Fe, New Mexico, December 2001).

[36] Osborne, J., And Rysdyk, R. Waypoint guidance for small UAVs in wind. In Proceedings of the American Institute of Aeronautics and Astronautics Infotech@Aerospace Conference (Arlington, VA, 2005).

[37] Papadakis, N. A., And Perakis, A. N. Deterministic minimal time vessel routing. Oper. Res. 38, 3 (1990), 426-438.

[38] Perakis, A. N., And Papadakis, N. A. Minimal time vessel routing in a timedependent environment. Transportation Sci. 23, 4 (1989), 266-276. 
[39] Philpotт, A. B. Stochastic optimization and yacht racing. In Applications of stochastic programming, vol. 5 of MPS/SIAM Ser. Optim. SIAM, Philadelphia, PA, 2005, pp. 315-336.

[40] Philpott, A. B., And Mason, A. Optimising yacht routes under uncertainty. In The 15th Cheasapeake Sailing Yacht Symposium (2001).

[41] Philpott, A. B., Sullivan, R. M., and Jackson, P. S. Yacht velocity prediction using mathematical programming. European Journal of Operational Research 67, 1 (May 1993), 13-24.

[42] Pontryagin, L. S., Boltyanskit, V. G., Gamkrelidze, R. V., And Mishchenko, E. F. The mathematical theory of optimal processes. Translated from the Russian by K. N. Trirogoff; edited by L. W. Neustadt. Interscience Publishers John Wiley \& Sons, Inc. New York-London, 1962.

[43] Reif, J. H., And Sun, Z. Movement planning in the presence of flows. Algorithmica 39, 2 (2004), 127-153.

[44] Rowe, N. C. Obtaining optimal mobile-robot paths with nonsmooth anisotropic cost functions using qualitative-state reasoning. The International Journal of Robotics Research 16, 3 (1997), 375-399.

[45] Rowe, N. C., And Ross, R. S. Optimal grid-free path planning across arbitrarily contoured terrain with anisotropic friction and gravity effects. IEEE Transactions on Robotics and Automation 6, 5 (October 1990), 540-553.

[46] Rysdyk, R. Course and heading changes in significant wind. Journal of Guidance, Control, and Dynamics 30, 4 (July - August 2007), 1168-1171.

[47] Souères, P., And Laumond, J.-P. Shortest paths synthesis for a car-like robot. IEEE Trans. Automat. Control 41, 5 (1996), 672-688.

[48] Stefanakis, E., And Kavouras, M. On the determination of the optimum path in space. In Spatial Information Theory - A Theoretical Basis for GIS (COSIT'95), A. U. Frank and W. Kuhn, Eds. Springer, Berlin, Heidelberg, 1995, pp. 241-257.

[49] Sun, Z., And Reif, J. H. On finding approximate optimal paths in weighted regions. J. Algorithms 58, 1 (2006), 1-32.

[50] Sun, Z., AND Rief, J. H. On finding energy-minimizing paths on terrains. IEEE Transactions on Robotics 21, 1 (February 2005), 102-114.

[51] Sussmann, H. J., And Tang, G. Shortest path for the Reeds-Shepp car: A worked out example of the use of geometric techniques in nonlinear optimal control. Tech. Rep. SYCON-91-10, Rutgers Center for Systems and Control, September 1991.

[52] Techy, L., And Woolsey, C. A. Minimum-time path planning for unmanned aerial vehicles in steady uniform winds. Journal of Guidance, Control, and Dynamics 32, 6 (November-December 2009), 1736-1746. 
[53] Widmayer, P., Wu, Y. F., And Wong, C. K. On some distance problems in fixed orientations. SIAM J. Comput. 16, 4 (1987), 728-746.

[54] Yu, C., Lee, J., and Munro-Stasiuk, M. J. Extensions to least-cost path algorithms for roadway planning. Int. J. Geogr. Inf. Sci. 17, 4 (2003), 361-376.

[55] Zermelo, E. Über das navigationsproblem bei ruhender oder veränderlicher windverteilung. Zeitschrift für Angewandte Mathematik und Mechanik 11, 2 (1931), 114-124. 